\title{
Nodal abdominal spread in adenocarcinoma of the cardia. Results of a multicenter prospective study
}

\author{
Giovanni de Manzoni ${ }^{1}$, Paolo Morgagni ${ }^{2}$, Franco Roviello ${ }^{3}$, Alberto Di Leo $^{1}$, Luca Saragoni ${ }^{4}$, \\ Daniele Marrelli ${ }^{3}$, Alfredo Guglielmi ${ }^{1}$, Alfonso Carli $^{3}$, Secondo Folli ${ }^{5}$, and Claudio Cordiano ${ }^{1}$ \\ ${ }^{1}$ Istituto di Semeiotica Chirurgica, 1a Divisione Clinicizzata di Chirurgia Generale, Università di Verona, Ospedale Civile Maggiore, Piazzale \\ Stefani, 1 Verona, Italy \\ ${ }^{2}$ Divisione di Chirurgia Generale, ${ }^{4}$ Servizio di Anatomia Patologica, ${ }^{5}$ Divisione di Chirurgia Toracica, Ospedale di Forli Forli, Italy \\ ${ }^{3}$ Istituto Scienze Chirurgiche, Università di Siena Siena, Italy
}

\begin{abstract}
:
Background. Precise knowledge of the abdominal nodal spread of cardia adenocarcinoma in relation to the depth of invasion of the tumor and its longitudinal extension may be very important for the surgeon as a guide in choosing the type of resection and lymphadenectomy.

Methods. The frequency of node metastases in each abdominal station of the first and second tier was prospectively studied in 101 patients with type II and III cardia cancer (defined as approved by the consensus conference held during the second International Gastric Cancer Conference in Munich in April, 1997) who underwent total gastrectomy with D2 lymphadenectomy during the period January 1994 to April 1998. Lymph nodes were retrieved immediately after operation by the surgeon and assigned to the appropriate station according to the classification of the Japanese Research Society for Gastric Cancer.

Results. In early gastric cancer, of both type II and type III, lymph node involvement was limited to the perigastric nodes of the upper half of the stomach and to the lymph node station of the celiac trunk. In advanced cancers, whether of type II or type III, there was a fairly high frequency of metastases to the perigastric nodes of the lower half of the stomach; there was also high frequency of metastases at $\mathrm{N} 2$ stations, without differences in frequency between pT2 and pT3 tumors (staged according to the classification of the Japanese Research Society for Gastric Cancer).

Conclusions. The results of our study provide evidence for the need to perform a total gastrectomy with D2 lymphadenectomy in all patients with advanced cardia cancer type II or type III. In early cancers, a less extensive resection (proximal gastrectomy) with D2 lymphadenectomy may be indicated.
\end{abstract}

Key words: cardia cancer, lymph node metastasis, surgery, extended lymphadenectomy

Offprint requests to: G. de Manzoni

Received for publication on Jul. 15, 1998; accepted on Oct. 22, 1998

\section{Introduction}

Recent studies reported in the literature have revealed that, despite the decreasing overall incidence of gastric cancer in Western countries, there has been an increase in adenocarcinomas localized in the proximal stomach, particularly at the level of the cardia [1-5].

There is a great deal of controversy regarding the definition, classification, and staging of this type of cancer [6,7]. Moreover, the extent of gastric and esophageal resection and the therapeutic value of extended lymphadenectomy are still much debated issues [8-12].

A thorough knowledge of the ways such tumors spread, both at organ and lymph node level, would undoubtedly be of great help as a guide to the surgeon in choosing the most appropriate type of resection and lymphadenectomy in these malignancies.

We therefore conducted a prospective multicenter study in order to assess the abdominal nodal spread of cardia adenocarcinoma in relation to the depth of invasion of the tumor and its longitudinal extension.

\section{Patients and methods}

\section{Method}

In this study, adenocarcinoma was defined according to the classification proposed by Siewert and recently approved during the consensus conference held during the second International Gastric Cancer Congress in Munich in April, 1997 [13].

Type I. Cardia adenocarcinoma extending to the esophagus with the center lying $1-5 \mathrm{~cm}$ above the anatomic esophago-gastric junction.

Type II. Cardia adenocarcinoma with the center lying $1 \mathrm{~cm}$ above to $2 \mathrm{~cm}$ below the anatomic esophago-gastric junction. 
Type III. Cardia adenocarcinoma extending to the stomach with the center lying $2-5 \mathrm{~cm}$ below the anatomic esophago-gastric junction.

Tumors were staged according to the classification of the Japanese Research Society for Gastric Cancer (JRSGC) [14], and the histologic classification was based on the Lauren criteria.

Perigastric lymph nodes (N1) were dissected from the excised specimens by the surgeons immediately after gastrectomy, assigned to the appropriate station according to the JRSGC classification, and sent for histologic examination. Among the lymph nodes on the greater curvature, those lying along the right gastro-epiploic artery were isolated from the lymph nodes of the upper half of the greater curvature (along the short vessels and the left gastro-epiploic artery).

To avoid errors due to difficulties in assigning the lymph nodes of the second compartment to the correct lymph node stations after en-bloc resection [15], the $\mathrm{N} 2$ nodes were subdivided by the surgeon during lymphadenectomy.

\section{Patients}

This prospective study included all patients suffering from type II or type III cardia adenocarcinoma who underwent total gastrectomy with distal esophagectomy, D2 abdominal lymphadenectomy (i.e. dissection of nodes in positions 1-11), and dissection at least of the lower mediastinal nodes, at the First Division of General Surgery, Verona University, at the Institute of Surgical Sciences, Siena University, and at the First Division of General Surgery, Forli Hospital, Italy during the period January 1994 to April 1998.

Reconstruction was performed in all patients by means of an intrathoracic or transhiatal Roux-en-Y esophagojejunostomy.

A superextended lymphadenectomy (D4, dissection extended to nodes in positions 12-16) was performed in 15 patients with cardial malignancies spreading to the stomach.

Patients with type I adenocarcinoma of the cardia $(n=33)$ were excluded from the study.

A total of 101 patients were recruited into the study (43 with type II and 58 with type III). The demographic data and tumor characteristics of these patients are summarized in Table 1.

\section{Results}

Seven patients died within the first 30 postoperative days (operative mortality rate, $6.9 \%$ ) and 25 patients $(24.7 \%)$ developed postoperative general and surgical complications, such as pneumonia, anastomotic leakage, pancreatic fistula.
Table 1. Main demographic and clinical characteristics of all 101 patients in our series who underwent total gastrectomy for cardia cancer between January 1994 and April 1998a

\begin{tabular}{lcc}
\hline & $\begin{array}{c}\text { Type II } \\
(n=43)^{\mathrm{c}}\end{array}$ & $\begin{array}{c}\text { Type III } \\
(n=58)^{\mathrm{c}}\end{array}$ \\
\hline $\begin{array}{l}\text { Mean age (years) (sd) } \\
\text { Sex }\end{array}$ & $60(11.7)$ & $62(11.2)$ \\
$\quad$ Male & 34 & 42 \\
Female & 9 & 16 \\
Depth of invasion & & \\
pT1 & $12(28)$ & $4(7)$ \\
pT2 & $13(30)$ & $20(34)$ \\
pT3 & $16(37)$ & $23(40)$ \\
pT4 & $2(4)$ & $11(19)$ \\
Borrmann classification & & \\
$\quad$ (advanced tumors) & $6(14)$ & $16(28)$ \\
B I & $19(44)$ & $25(43)$ \\
B II & $6(14)$ & $11(19)$ \\
B III & 0 & $2(3)$ \\
B IV & & $31(53)$ \\
Lauren classification & $32(75)$ & $20(35)$ \\
Intestinal type & $7(16)$ & $7(12)$ \\
Diffuse type & $4(9)$ & $4(7)$ \\
Mixed type & $4(9)$ & $20(35)$ \\
Histological grading & $19(44)$ & $12(20)$ \\
Well differentiated & $4(9)$ & \\
Moderately differentiated & & \\
Poorly differentiated & & \\
Not classified & (1) & \\
\hline
\end{tabular}

a Institutions in the series were: First Division of General Surgery, Verona University; Institute of Surgical Sciences, Siena University; and First Division of General Surgery, Forli Hospital, Italy

${ }^{\mathrm{b}}$ See text for definitions of type II and type III

c Values in parentheses are percentages

${ }^{\mathrm{d}}$ Staged according to the classification of the Japanese Research Society for Gastric Cancer [14]

The total number of lymph nodes dissected in the 101 patients, was 3114. The mean number of nodes dissected per patient was $31.2 \pm 14.7$ (range, $10-108$ ), and the mean number of metastatic nodes was $6.2 \pm 6.7$ (range, 0-39).

The frequency of lymph node involvement in relation to depth of tumor invasion is given in Table 2 for the pure cardial tumors (type II) and in Table 3 for the subcardial tumors with invasion of the cardia (type III). As shown, there was a high frequency of patients with lymph node metastases in both types of malignancies ( $70 \%$ of patients with type II tumors and $83 \%$ of patients with type III tumors). The frequency of lymph node metastases in type II adenocarcinomas appeared to be very high right from stage pT1, whereas in type III pT1 tumors, the lymph node spread of the malignancies closely resembled that of early stomach cancers at other gastric sites.

No significant differences in frequency of lymph node metastases could be discerned between pT2 and pT3 tumors among either the type II or type III 
adenocarcinomas. As postulated by Siewert et al. [16], in gastric tumors of the upper third of the stomach this may be because $\mathrm{T} 2$ tumors at this site involve the full thickness of the gastric wall in most instances, thus resembling T3 malignancies, despite there being no

Table 2. Frequency of lymph node metastases in relation to depth of tumor invasion in the 43 patients with type II adenocarcinoma of the cardia

\begin{tabular}{lcccc}
\hline & $\mathrm{pN}^{\mathrm{a}}$ & $\mathrm{pN}+{ }^{\mathrm{a}}$ & $\mathrm{pN} 1^{\mathrm{a}}$ & $\mathrm{pN} 2^{\mathrm{a}}$ \\
\hline pT1 $(n=12)$ & $8(67)$ & $4(33)$ & $3(25)$ & $1(8)$ \\
pT2 $(n=13)^{\mathrm{b}}$ & $2(15)$ & $11(85)$ & $5(38)$ & $6(46)$ \\
pT3 $(n=16)$ & $3(19)$ & $13(81)$ & $5(31)$ & $8(50)$ \\
pT4 $(n=2)$ & 0 & $2(100)$ & 0 & $2(100)$ \\
Total & $13(30)$ & $30(70)$ & $13(30)$ & $17(40)$ \\
\hline
\end{tabular}

${ }^{a}$ Values in parentheses are percentages

${ }^{\mathrm{b}}$ Patients with transmural growth and invasion of the subserosa or perivisceral fat

Table 3. Frequency of lymph node metastases in relation to depth of tumor invasion in 58 patients with type III adenocarcinoma of the cardia

\begin{tabular}{lllrr}
\hline & pN0 & \multicolumn{1}{c}{$\mathrm{pN}^{\mathrm{a}}$} & \multicolumn{1}{c}{$\mathrm{pN}^{\mathrm{a}}$} & \multicolumn{1}{c}{$\mathrm{pN}^{\mathrm{a}}$} \\
\hline pT1 $(n=4)$ & 4 & 0 & 0 & 0 \\
pT2 $(n=20)^{\mathrm{b}}$ & $3(15)$ & $17(85)$ & $9(45)$ & $8(40)$ \\
pT3 $(n=23)$ & $3(13)$ & $20(87)$ & $10(43)$ & $10(43)$ \\
pT4 $(n=11)$ & 0 & $11(100)$ & $3(27)$ & $8(73)$ \\
Total & $10(17)$ & $48(83)$ & $22(38)$ & $26(45)$ \\
\hline
\end{tabular}

${ }^{a}$ Values in parentheses are percentages

${ }^{b}$ Four patients with invasion of the muscularis propria and 16 with transmural growth and invasion of the subserosa or perivisceral fat without perforation of the peritoneum involvement of the tunica serosa, which, moreover, is present at cardial level only on the anterior wall.

Below we provide detailed analysis of the tumor spread to the individual stations in relation to the type of malignancy examined.

Type II adenocarcinoma. The nodal spread of type II cardia adenocarcinoma to each abdominal lymph node station of the first and second tiers is shown in Fig. 1.

All the pT2 specimens analyzed showed infiltration of the subserosal layer or of the perivisceral fat; the only two pT4 specimens examined were included in the analysis of the pT3 group.

In the early tumors, the only lymph node stations affected by metastases were the left paracardial ( $8 \%$ of cases) and lesser curvature (17\% of cases) stations in the N1 group and the celiac trunk station (13\% of cases) in the $\mathrm{N} 2$ group.

In the pT2 tumors, in addition to a predictably high frequency of lymph node metastases affecting the perigastric nodes of the upper half of the stomach (right paracardial $50 \%$, left paracardial $31 \%$, lesser curvature $78 \%$ ), also worthy of note is the presence of metastases affecting the lymph nodes along the right gastroepiploic artery ( $8 \%$ of cases) and the suprapyloric lymph nodes (13\% of cases). There were, however, no metastases affecting the infrapyloric lymph nodes. In the N2 lymph node station group, on the other hand, there was a marked lack of metastases to the lymph nodes of the splenic hilus and artery.

In the pT3-pT4 carcinomas, practically all the perigastric stations were found to harbor metastases. Particularly important was the involvement of the lymph nodes of the right gastroepiploic artery $(18 \%$ of cases) and of the infrapyloric lymph nodes $(25 \%$ of cases), while metastases to the suprapyloric lymph

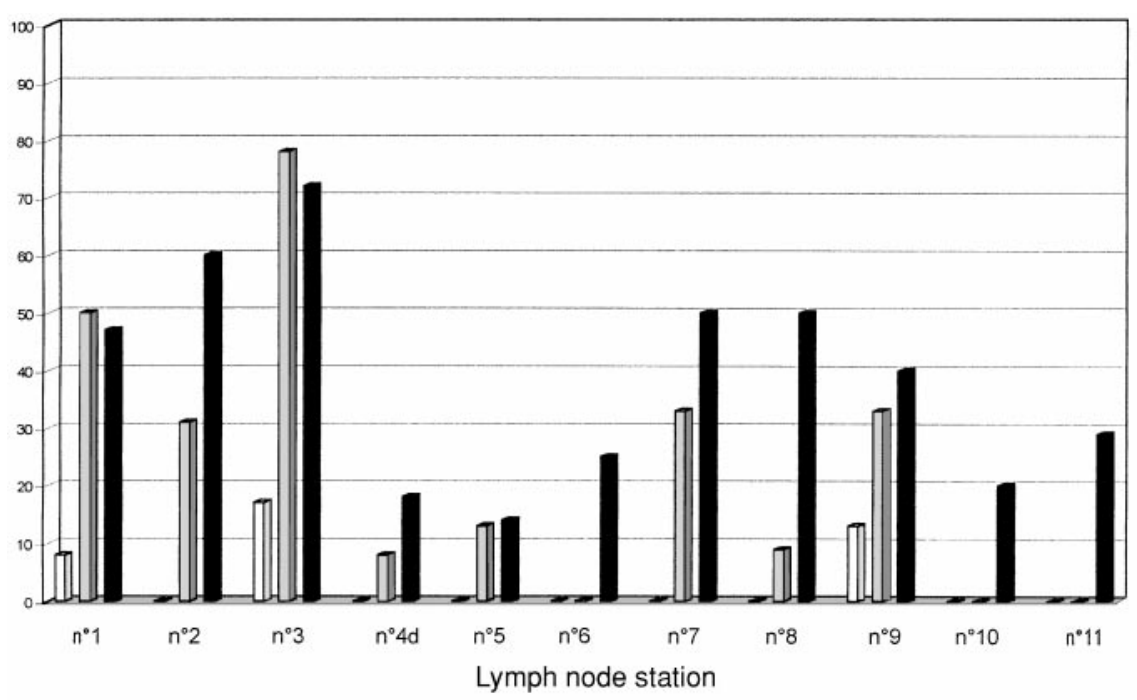

Fig. 1. Frequency of metastasis to each lymph node station in patients with type II adenocarcinoma of the cardia. White bars, pT1 tumors; gray bars, pT2 tumors; black bars, pT3-pT4 tumors. See text for definition of type II. pT and lymph node stations classified according to the Japanese Research Society for Gastric Cancer Criteria [14] 


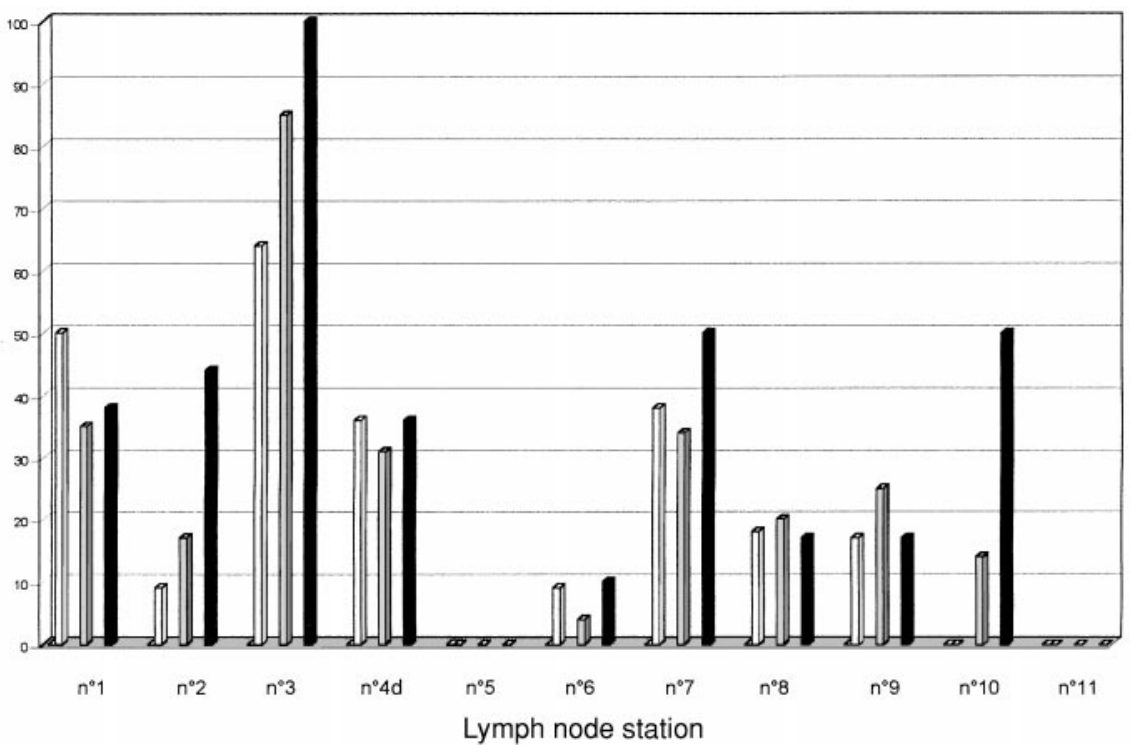

Fig 2. Frequency of metastasis to each lymph node station in patients with type III adenocarcinoma of the cardia. White bars, pT2 tumors; gray bars, pT3 tumors; black bars, pT4 tumors. See text for definition of type III nodes were detected in $14 \%$ of cases. As regards the N2 stations, a substantial percentage of metastases affected the splenic hilus $(20 \%)$ and artery $(29 \%)$.

Type III adenocarcinoma. The nodal spread in type III adenocarcinomas is illustrated in Fig. 2.

No lymph node metastases were detected in pT1 tumors. The number of cases observed, however, was very small $(n=4)$, and the zero metastasis rate cannot therefore be regarded as a definitive finding, although it is, all the same, indicative of very limited nodal spread.

In the stage pT2 tumors, as far as the perivisceral stations were concerned, there was a high frequency of metastases to the lymph nodes along the right gastroepiploic artery (36\%), with by no means negligible metastatic spread to the suprapyloric lymph nodes as well $(9 \%)$. In the $\mathrm{N} 2$ group, we should stress the frequency of involvement of the hepatic artery (18\%), accompanied, in one case out of six, by involvement of the lymph nodes along the anterior surface of the hepatoduodenal ligament (data not indicated in the graph). The para-aortic station harbored metastases in one of five pT2 patients who had D4 lymphadenectomy.

In the pT3 carcinomas, all the perigastric stations, with the sole exception of the suprapyloric station, were affected by metastases. In particular, the frequency of metastases was $31 \%$ in the right gastroepiploic artery station and $4 \%$ in the infrapyloric station. Also worthy of note is the presence, in the $\mathrm{N} 2$ group, of metastases affecting the splenic hilus (14\% of cases).

In the six patients in whom a superextended D4 lymphadenectomy was performed, the hepatoduodenal ligament station was unaffected by metastases, whereas the para-aortic lymph nodes presented metastastic involvement in one patient.
In the pT4 tumors, too, the only perigastric station which was metastasis-free was the suprapyloric station, while metastases to the right gastroepiploic artery station were present in $36 \%$ of cases and to the infrapyloric lymph nodes in $10 \%$. In the $\mathrm{N} 2$ group, there was a particularly high frequency of metastatic spread to the lymph nodes of the splenic hilus $(50 \%)$. In the four patients who had D4 lymphadenectomy, the hepatoduodenal ligament station was positive in one and the para-aortic station was in two positive.

\section{Discussion}

The data reported in the literature regarding the spread of adenocarcinoma of the cardia are scanty and hard to compare, both because of the frequent pooling of squamous-cell carcinoma of the distal esophagus and cardia adenocarcinoma [17], and because of the lack of any overall consensus as to the definition and staging of the latter type of tumor [18]. Abdominal nodal spread in cancer of the cardia has been studied in a number of recent reports which, however, present no analysis of the involvement of the individual abdominal lymph node stations in relation to the $\mathrm{pT}$ stage and longitudinal extension of the tumors [18-21]. Precise knowledge of these data may be very important for the surgeon as a guide in choosing the type of resection and lymphadenectomy. This prospective multicenter study was undertaken, therefore, with the aim of collecting, within a short period of time, a sizeable number of patients in whom the abdominal nodal spread could be studied thoroughly for each regional lymph node station and related not only to the depth of tumor invasion 
but also to the type of longitudinal extension, as described in the Siewert classification.

The main findings of the present study are:

(1) in early malignancies, of both type II and type III, abdominal lymph node involvement is limited and confined to the perigastric lymph nodes of the upper half of the stomach (right paracardial and lesser curvature) and the lymph nodes of the celiac trunk.

(2) In advanced adenocarcinomas, whether of type II or type III, we found a fairly high frequency of metastases to the perigastric stations of the lower half of the stomach (lymph nodes of the distal portion of the greater curvature, infrapyloric and suprapyloric lymph nodes). As regards the second-tier stations, the frequency of metastases, as also reported by other investigators, was high in both types of adenocarcinoma. Moreover, the patients who had had D4 lymphadenectomy presented metastases to the lymph nodes of the hepatoduodenal ligament and to the para-aortic lymph nodes in all pT stages (pT2, pT3, and pT4).

(3) There were no differences in frequency of overall lymph node metastases, either at the N1 or N2 stations, between pT2 and pT3 tumors in either of the types of cardia adenocarcinoma studied. The uniformity of the nodal spread of these two types of tumors (pT2 and pT3) is even more marked if we eliminate from the pT2 group the very few patients $(n=4)$ with invasion of the tunica muscularis alone, which invasion is present only in type III malignancies.

We can conclude by saying that, the results of this study provide evidence for the need to perform a total gastrectomy in all patients with advanced type II or III adenocarcinomas of the cardia, to avoid leaving infiltrated lymph nodes along the greater curvature of the stomach in a substantial percentage of these patients. An exception to this rule of thumb may be in those individuals with early malignancies in whom noninvolvement of the lymph node stations in the lower half of the stomach may allow a less extensive resection (proximal gastrectomy), as suggested by other authors [22,23].

As far as lymphadenectomy is concerned, the high percentage of involvement of second-tier lymph node stations makes the performance of D2 lymphadenectomy mandatory. Indeed, in our experience [24], as well as that of other groups in the West $[25,26]$, the mortality and morbidity rates after $\mathrm{D} 2$ dissection were similar to or even lower than those after the limited D1 dissection in the Dutch [27] and British [28] trials. Furthermore, the presence of metastases to para-aortic lymph nodes in $17 \%-25 \%$ of our patients with a D4 lymphadenectomy suggests that the degree of involvement and the precise role of this station should be examined more thoroughly with studies in larger D4 patient samples.
Lastly, our results reveal a substantial measure of uniformity of nodal spread in tumors that infiltrate the full thickness of the wall of the organ regardless of the absence (pT2) or presence (pT3) of serosal invasion. This finding, as we see it, may lead to modifications of the TNM staging in cardial tumors and to grouping these pT2-pT3 tumors together in a single group, as has been proposed by Skinner et al. for esophageal cancer [29].

\section{References}

1. Kampschöer GH, Nakajima M, van de Velde CJH. Changing patterns in gastric adenocarcinoma. Br J Surg 1989;76:914-16.

2. Golematis B, Tzardis P, Hatzikostas P, Papadimitriou K, Haritopolus N. Changing pattern of distribution of carcinoma of the stomach. Br J Surg 1990;77:63-4.

3. Blot WJ, Devesa SS, Kneller RW, Fraumeni JF Jr. Rising incidence of adenocarcinoma of the esophagus and gastric cardia. JAMA 1991;265:1287-9.

4. Craanen ME, Dekker W, Blok P, Ferwerda J, Tytgat GNJ. Time trends in gastric carcinoma: Changing patterns of type and location. Am J Gastroenterol 1992;87:572-9.

5. Pera M, Cameron AJ, Trastek VF, Carpenter HA, Zinsmeister AR. Increasing incidence of adenocarcinoma of esophagus and esophagogastric junction. Gastroenterology 1993;104:510-13.

6. Husemann B. Cardia carcinoma considered as a distinct clinical entity. Br J Surg 1989;76:136-9.

7. Steup WH, De Leyn P, Deneffe G, Van Raemdonck D, Coosemans W, Lerut T. Tumors of the esophagogastric junction. Long-term survival in relation to the pattern of lymph node metastasis and a critical analysis of the accuracy or inaccuracy of pTNM classification. J Thorac Cardiovasc Surg 1996;111:8595

8. Skinner DB, Ferguson MK, Soriano A, Little AG, Staszak VM. Selection of operation for esophageal cancer based on staging. Ann Surg 1986;204:391-401.

9. DeMeester TR, Zaninotto G, Johansson KK. Selective therapeutic approach to cancer of the esophagus and cardia. J Thorac Cardiovasc Surg 1988;95:42-54.

10. Stipa S, Di Giorgio A, Ferri M. Surgical treatment of adenocarcinoma of the cardia. Surgery 1992;111:386-93.

11. Lerut T, DeLeyn P, Coosmans W, Van Raemdonck D, Scheys I, Lesaffre E. Surgical strategies in esophageal carcinoma with emphasis on radical lymphadenectomy. Ann Surg 1992;216:58390.

12. Hagen JA, Peters JH, DeMeester TR. Superiority of extended en bloc esophagogastrectomy for carcinoma of the lower esophagus and cardia. J Thorac Cardiovasc Surg 1993;106:850-9.

13. Siewert JR, Hölscher AH, Becker K, Gössner W. Kardiacarcinom: Versuch einer therapeutisch relevanten Klassifikation. Chirurg 1987;58:25-32.

14. Japanese Research Society for Gastric Cancer. Japanese classification of gastric cancer. 1st English edition. Tokyo: Kanehara; 1995.

15. Bunt TNG, Bonenkamp HJ, Hermans J, van de Velde CJH, Arends J-W, Fleuren G, Brujin JA. Factors influencing noncompliance and contamination in a randomized trial of Western (r1.) versus Japanese (r2.) type surgery in gastric cancer. Cancer 1994;73:1544-51.

16. Siewert JR, Bottcher K, Stein HJ, Roder JD, Busch R. Problems of proximal third carcinoma. World J Surg 1995;19:523-31.

17. Lozac'h P. Cancer du bas oesophage et du cardia. J Chir 1997;134: 195-6. 
18. Griffin SM, Chung SCS, Woods SDS, Li AKC. Adenocarcinoma of the cardia: Treatment by thoracoabdominal R3 radical gastrectomy. Br J Surg 1990;77:937-9.

19. Clark GWB, Peters JH, Ireland AP, Ehsan A, Hagen JA, Kiyabu MT, et al. Nodal metastasis and sites of recurrence after en bloc esophagectomy for adenocarcinoma. Ann Thorac Surg 1994;58:646-54.

20. Wang L-S, Wu C-W, Hsieh M-J, Fahn H-J, Huang M-H, Chien K-Y. Lymph node metastasis in patients with adenocarcinoma of gastric cardia. Cancer 1993;71:1948-53.

21. Hsu CP, Wu CC, Chen CY, Hsu NY, Hsia JY, Wang PY. Clinical experience in radical lymphadenectomy for adenocarcinoma of the gastric cardia. J Thorac Cardiovasc Surg 1997;114:54451.

22. Kitamura K, Yamaguchi T, Nishida S, Yamamoto K, Ichikawa D, Okamoto $\mathrm{K}$, et al. The operative indications for proximal gastrectomy in patients with gastric cancer in the upper third of the stomach. Surg Today 1997, 27:993-8.

23. Harrison LE, Schwartz RE, Karpeh MS, Brennan MF. Total gastrectomy is not necessary for proximal gastric cancer. In Siewert JR, Roder JD, editors. Progress in gastric cancer research 1997. Vol 2. Bologna; Monduzzi Editore, 1997:929-34.
24. de Manzoni G, Verlato G, Guglielmi A, Laterza E, Genna M, Cordiano C. Prognostic significance of lymph node dissection in gastric cancer. Br J Surg 1996;83:1604-7.

25. Sue-Ling HM, Johnston D, Martin IG. Gastric cancer: A curable disease in Britain. BMJ 1993;307:591-6.

26. Siewert JR, Bottcher K, Roder JD, Busch R, Hermanek P, Meyer HJ and the German Gastric Carcinoma Study Group. Prognostic relevance of systematic lymph node dissection in gastric carcinoma. Br J Surg 1993;80:1015-18.

27. Bonenkamp JJ, Songun I, Hermans J, Sasako M, Welvaart K, Plukker JTM, et al. Randomised comparison of morbidity after D1 and D2 dissection for gastric cancer in 996 Dutch patients. Lancet 1995;345:745-8.

28. Cuschieri A, Fayers P, Fielding J, Craven J, Bancewicz J, Joypaul V, Cook P. Postoperative morbidity and mortality after D1 and D2 resections for gastric cancer: Preliminary results of the MRC randomised controlled surgical trial. Lancet 1996;347:995-9.

29. Skinner DB, Dowlatshahi KD, DeMeester TR. Potentially curable cancer of the esophagus. Cancer 1982;50:2571-5. 